\title{
(2) OPEN ACCESS \\ Improvement in time to treatment, but not time to diagnosis, in patients with mucopolysaccharidosis type I
}

\author{
Roberto Giugliani 지, ${ }^{1}$ Nicole Muschol, ${ }^{2}$ Hillary A. Keenan, ${ }^{3}$ Mark Dant, ${ }^{4}$ \\ Joseph Muenzer ${ }^{5}$
}

'Departamt of Genetics, UFRGS, Medical Genetics Service, HCPA, Porto Alegre, Brazil

2Department of Pediatrics, International Center for Lysosomal Disorders (ICLD) University Medical Center Hamburg-Eppendorf, Hamburg, Germany

${ }^{3}$ Biostatistics and Epidemiology, Rare Disease Registries, Global Medical Affairs, Sanofi Genzyme, Cambridge, Massachusetts, USA ${ }^{4}$ The Ryan Foundation and EveryLife Foundation for Rare Diseases, Washington, DC, USA ${ }^{5}$ Department of Pediatrics, University of North Carolina at Chapel Hill, Chapel Hill, North Carolina, USA

\section{Correspondence to}

Professor Roberto Giugliani, Departamento de Genetica, Universidade Federal do Rio Grande do Sul Instituto de Biociencias, Porto Alegre, Brazil; rgiugliani@hcpa.edu.br

Received 21 February 2020 Revised 10 September 2020 Accepted 9 October 2020 Published Online First

2 November 2020

\section{ABSTRACT}

Objective Early diagnosis and treatment initiation are important factors for successful treatment of mucopolysaccharidosis type I (MPS I). The purpose of this observational study was to assess whether age at diagnosis and time to first treatment for individuals with MPS I have improved over the last 15 years.

Study design Data from the MPS I Registry

(NCT00144794) for individuals with attenuated or severe disease who initiated therapy with laronidase enzyme replacement therapy (ERT) and/or hematopoietic stem cell transplantation (HSCT) between 1 January 2003 and 31 December 2017 were included.

Results Data were available for 740 individuals with attenuated ( $n=291$ ) or severe ( $n=424$ ) MPS I (unknown $n=25$ ). Median age at diagnosis for attenuated disease did not change over time and ranged between 4.5 and 6 years of age while the median duration from diagnosis to first ERT decreased from 5.6 years before/during 2004 to 2.4 months in 2014-2017. For severe MPS I treated with HSCT, median age at diagnosis was less than 1 year and median time to first treatment was less than 3 months throughout the 15-year observation period.

Conclusions Times to diagnosis and HSCT initiation for individuals with severe MPS I were consistent over time. For individuals with attenuated MPS I, the time to ERT initiation after diagnosis has improved substantially in the last 15 years, but median age at diagnosis has not improved. Efforts to improve early diagnosis in attenuated MPS I are needed to ensure that patients receive appropriate treatment at the optimal time.

\section{INTRODUCTION}

Mucopolysaccharidosis type I (MPS I) results from deficient $\alpha$-L-iduronidase, a lysosomal enzyme responsible for glycosaminoglycans (GAGs) dermatan and heparan sulfate metabolism. ${ }^{1}$ MPS I is a pan-ethnic, autosomal-recessive disease, with an incidence that varies across regions and is estimated as $1 / 100000$ live births. ${ }^{2}$ Disease phenotypes range from severe (Hurler syndrome) to attenuated (Hurler-Scheie and Scheie syndromes) depending on presence or absence of central nervous system (CNS)/neurocognitive involvement and rate of disease progression. ${ }^{145}$ If untreated, MPS I results in significant disease burden and disability, with premature death possible from respiratory and cardiac disease. ${ }^{2}$

Treatment options include haematopoietic stem cell transplantation (HSCT) for severe disease, and

\section{What is already known on this topic?}

- Mucopolysaccharidosis type I (MPS I) is an inherited lysosomal storage disease where affected individuals experience significant disease burden, disability and premature death.

- Early treatment with enzyme replacement therapy and/or haematopoietic stem cell transplantation can reduce/halt disease progression and improve outcomes. However, diagnosis, and consequently the start of therapy, is often delayed, particularly for patients with attenuated phenotypes.

\section{What this study adds?}

- Our analyses show that while time to diagnosis for attenuated MPS I is still delayed, time to treatment has improved significantly over the 15 years since enzyme replacement therapy was introduced.

enzyme replacement therapy (ERT) with laronidase (recombinant human $\alpha$-L-iduronidase; Aldurazyme) for treatment of non-CNS manifestations of MPS I ${ }^{6-9}$ and in the peritransplant period for severe disease. ${ }^{10}$ Treatment outcomes are influenced both by disease severity and age at treatment initiation. ${ }^{11} 12$ Early treatment considerably improves patient outcomes during long-term therapy and is crucial to reduce or halt disease progression before irreversible damage occurs. ${ }^{12-16}$ However, diagnosis of MPS I is often delayed, particularly for patients with attenuated phenotypes, resulting in delayed introduction of treatment. ${ }^{17-23}$

Data from the MPS I Registry were analysed to understand trends in diagnosis and treatment in individuals with severe or attenuated MPS I since introduction of ERT and to identify potential factors influencing delays in times to diagnosis and/or treatment (eg, regional differences; disease severity). These data will help us to understand where improvements are still needed to decrease the time to diagnosis and treatment in order to ensure that individuals with MPS I receive the appropriate treatments before significant onset of disease. 


\section{METHODS}

\section{Registry}

The MPS I Registry (NCT00144794) is a voluntary, observational global longitudinal database established to capture longterm data to help understand the natural history, standards of care and treatment outcomes of individuals with MPS I. ${ }^{24}$ Individuals are enrolled in the MPS I Registry by their clinician and data are collected both retrospectively and prospectively as previously described. ${ }^{24}$ The Registry is overseen and directed by an independent Board of Advisors comprised of physicians who are experts in the care of MPS disorders and is sponsored by Sanofi Genzyme (Cambridge, MA). All participants (patients or caregivers) provide written informed consent for use of anonymised data.

\section{Participants}

Observations for individuals in the Registry with either severe or attenuated MPS I were included if MPS I treatment was received between 1 January 2003 and 31 December 2017. For an observation to be included, valid dates for diagnosis and start of treatment were required.

\section{Data management and analysis}

Current, primary treatments for analysis included HSCT or ERT (not including peritransplant ERT). For patients with more than one HSCT, first HSCT date was used as start of primary treatment date. Patients with ERT as primary treatment did not receive HSCT. Those for whom HSCT was primary treatment and peritransplant ERT was received, ERT was not counted as primary treatment.

ERT with laronidase became available in 2003, and the MPS I Registry also began enrolling participants in 2003. Data were stratified by MPS I phenotype into year intervals at first primary MPS I therapy. Four intervals were selected for data analysis: in/before 2004 to coincide with initiation of the Registry and laronidase ERT and groupings of 2005-2008, 2009-2013 and after 2013-2017 to have similar time periods covered by each group. Data were also stratified by region where laronidase is approved for commercial use, and presented when sufficient patient numbers for comparisons were available.

All data were analysed as entered by the clinical site. Descriptive statistics include number, percentage, mean, median, SD and IQRs.

\section{RESULTS}

Among all participants in the MPS I Registry from January 2003 to December 2017, 740 had data for age at diagnosis and age at treatment. The distribution by region and timing of enrolment for individuals with treatment for MPS I are shown in table 1, with most participants enrolled before 2009. Distribution of participants was evenly divided between Europe (45.8\%) and North America (44.5\%) with a small percentage of individuals from Latin America (7\%) or Asia Pacific (2.7\%).

Table 1 Regional distribution of registry participants with treatment history

\begin{tabular}{lcll}
\hline & $\begin{array}{l}\text { Participants } \\
\mathrm{n}=\mathbf{7 4 0}\end{array}$ & \multicolumn{1}{l}{ Enrolment } & \\
\cline { 3 - 4 } & & $\mathbf{2 0 0 3 - 2 0 0 9}$ or before & After 2009 \\
\hline Europe, $\mathrm{n}(\%)$ & $339(45.8)$ & $234(51.3)$ & 284 \\
\hline North America, $\mathrm{n}(\%)$ & $329(44.5)$ & $187(41.0)$ & $105(37.0)$ \\
\hline Latin America, $\mathrm{n}(\%)$ & $52(7.0)$ & $32(7.0)$ & $142(50.0)$ \\
\hline Asia Pacific, $\mathrm{n}(\%)$ & $20(2.7)$ & $3(0.7)$ & $20(7.0)$ \\
\hline
\end{tabular}

Table 2 Demographic and clinical characteristics

\begin{tabular}{|c|c|c|}
\hline & \multicolumn{2}{|c|}{$\begin{array}{l}\text { Individuals with treatment history } \\
\mathrm{n}=740\end{array}$} \\
\hline \multicolumn{3}{|l|}{ Gender $n(\%)$} \\
\hline Female & \multicolumn{2}{|l|}{$362(48.9)$} \\
\hline Male & \multicolumn{2}{|l|}{$378(51.1)$} \\
\hline \multicolumn{3}{|l|}{ MPS I phenotype n (\%) } \\
\hline Severe & \multicolumn{2}{|l|}{$424(57.3)$} \\
\hline Attenuated & \multicolumn{2}{|l|}{$291(39.3)$} \\
\hline \multirow[t]{3}{*}{ Undetermined/missing } & \multicolumn{2}{|l|}{$25(3.4)$} \\
\hline & \multicolumn{2}{|l|}{ Primary treatment } \\
\hline & $\begin{array}{l}\text { ERT } \\
(n=471)\end{array}$ & $\begin{array}{l}\mathrm{HSCT} \\
\mathrm{n}=269\end{array}$ \\
\hline \multirow[t]{5}{*}{ Age at diagnosis, year median (IQR) } & $3.4(1.2,6.6)$ & $0.9(0.6$ to 1.2$)$ \\
\hline & \multicolumn{2}{|c|}{ Age distribution, year $\mathbf{n}(\%)$} \\
\hline & $<5310(65.8)$ & $<1124(46.1)$ \\
\hline & $5-<1093(19.7)$ & $1-<2126(46.8)$ \\
\hline & $\geq 1068$ (14.4) & $\geq 219(7.1)$ \\
\hline \multirow{5}{*}{$\begin{array}{l}\text { Age at start of treatment, year median } \\
\text { (IQR) }\end{array}$} & $6.1(2.8$ to 12.8$)$ & $1.1(0.7$ to 1.4$)$ \\
\hline & \multicolumn{2}{|c|}{ Age distribution, year $\mathrm{n}(\%)$} \\
\hline & $<5203(43.1)$ & $<124(46.1)$ \\
\hline & $5-<10113(24.0)$ & $1-<2126(46.8)$ \\
\hline & $\geq 10155$ (32.9) & $\geq 219(7.1)$ \\
\hline \multicolumn{3}{|l|}{ HSCT treatment history } \\
\hline \# HSCT & & 305 \\
\hline
\end{tabular}

ERT, enzyme replacement therapy; HSCT, haematopoietic stem cell transplant.

Table 2 shows the demographic and clinical characteristics overall and stratified by first primary treatment (ERT or HSCT). Males and females were equally represented. In the entire cohort, 424/740 (57.3\%) were categorised with severe disease and 291/740 (39.3\%) with attenuated disease, whereas 25/740 (3.4\%) did not have a phenotype assigned.

HSCT treatment trends over time for pretreatment regimens and transplant type and peritransplant ERT are shown in figure 1. The trends generally follow the changes in HSCT recommendations and guidelines that evolved with time and experience. For the most recent time interval, cord blood was the most common source of stem cells (figure 1A) reported in the Registry, and pretransplant conditioning with fludarabine, busulfan and/or antithymocyte globulin/serum or antilymphocyte globulin/serum were the most common regimens. Peritransplant ERT was used pretransplant and/or post-transplant in increasing numbers of patients over time (figure 1B).

\section{Age at MPS I diagnosis}

Ages at diagnosis stratified by year interval of first primary therapy and region are shown for individuals with severe or attenuated MPS I for whom ERT was first primary therapy in figure 2 and for HSCT as first primary therapy for individuals with severe MPS I in figure 3.

Median ages at diagnosis for individuals with attenuated disease did not change appreciably over time since introduction of ERT and varied between 4.5 and 6 years of age (figure 2A). Among all individuals with severe disease receiving ERT as first primary therapy (these individuals did also not receive HSCT), ages at diagnosis remained fairly stable and varied between 1 and 2 years of age (figure 2A). Medians (IQR) for the intervals before/during 2004, 2005-2008, 2009-2013 and after 2013 to 2017 were $5.3(3.4,9.4), 5.0(3.0,10.2), 4.5(3.3,8.0)$ and 6.0 $(4.2,21.8)$ years, respectively, for the attenuated group and 1.2 $(0.6,2.1), 1.4(0.7,2.5), 2.0(0.7,3.9)$ and $1.1(0.7,2.4)$ years, 
A.

Type of HSCT

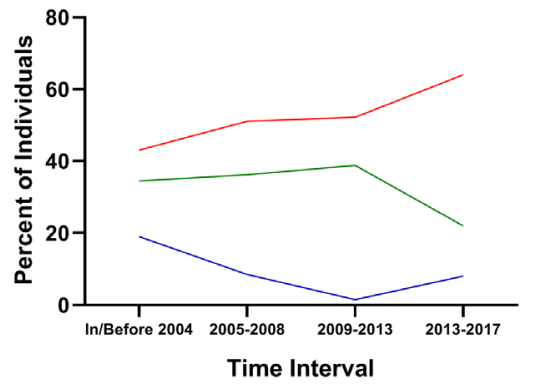

- Bone Marrow

- Cord Blood

- Peripheral Blood

B.

Peri-transplant ERT

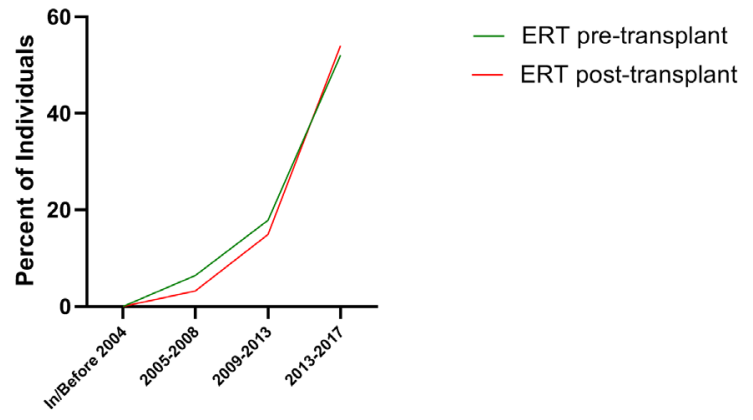

Time Interval

Figure 1 Characteristics of HSCT over time for individuals enrolled in the MPS I Registry. The type of transplant performed showing the source of stem cells is shown in (A). Use of ERT either before or immediately after transplant is shown in (B). ERT, enzyme replacement therapy; HSCT, haematopoietic stem cell transplants; MPS I, mucopolysaccharidosis type I.

respectively, for the severe group. Results were similar for individuals from North America (figure 2B) or Europe (figure 2C).

Median ages at diagnosis for individuals with severe disease receiving HSCT as first primary therapy were 0.8 or 0.9 years for the four time intervals (figure 3A). Median (IQR) for the intervals before/during 2004, 2005-2008, 2009-2013 and after 2013 to 2017 were $0.8(0.6,1.1), 0.9(0.7,1.2), 0.8(0.5,1.1)$ and $0.9(0.6,1.2)$ years, respectively, and in general, were lower than those for individuals with severe disease receiving ERT as first therapy. Similar results were noted regionally for individuals with severe disease (figure 3B,C).

\section{Time to first primary treatment}

Times from diagnosis to first primary treatment stratified by phenotype, year interval of first primary therapy and region are shown for ERT as first primary therapy in figure 4 and for HSCT as first primary therapy in figure 5 .

The median durations from diagnosis to first ERT for all individuals with attenuated or severe MPS I decreased over time since introduction of ERT (figure 4A). Medians varied from 5.6 years for individuals with attenuated disease before or during 2004 to 2.4 months in the most recent time interval for both severe and attenuated disease. Medians (IQR) for the intervals before/during 2004, 2005-2008, 2009-2013 and after 2013 to 2017 were $5.6(1.9,11.9), 1.9(0.4,5.2), 0.2(0.1,0.8)$ and 0.2 $(0.1,0.9)$ years, respectively, for the attenuated group and 2.0 $(0.6,4.8), 0.8(0.1,2.0), 0.2(0.1,0.4)$ and $0.2(0.1,0.5)$ years, respectively, for the severe group. When the data were stratified by region, there was a greater duration from diagnosis to

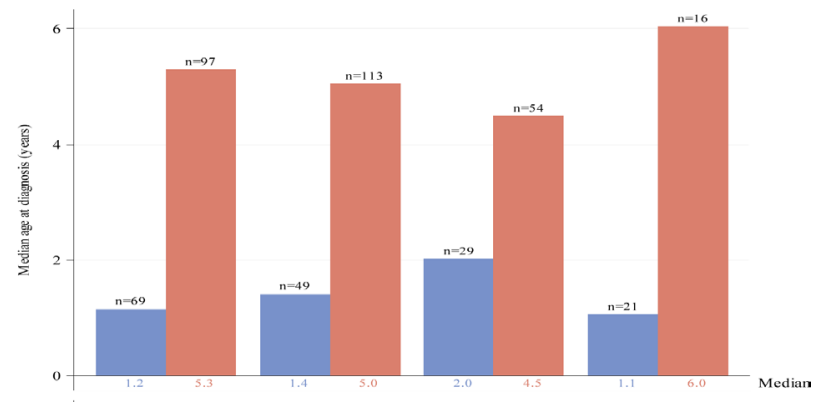

B

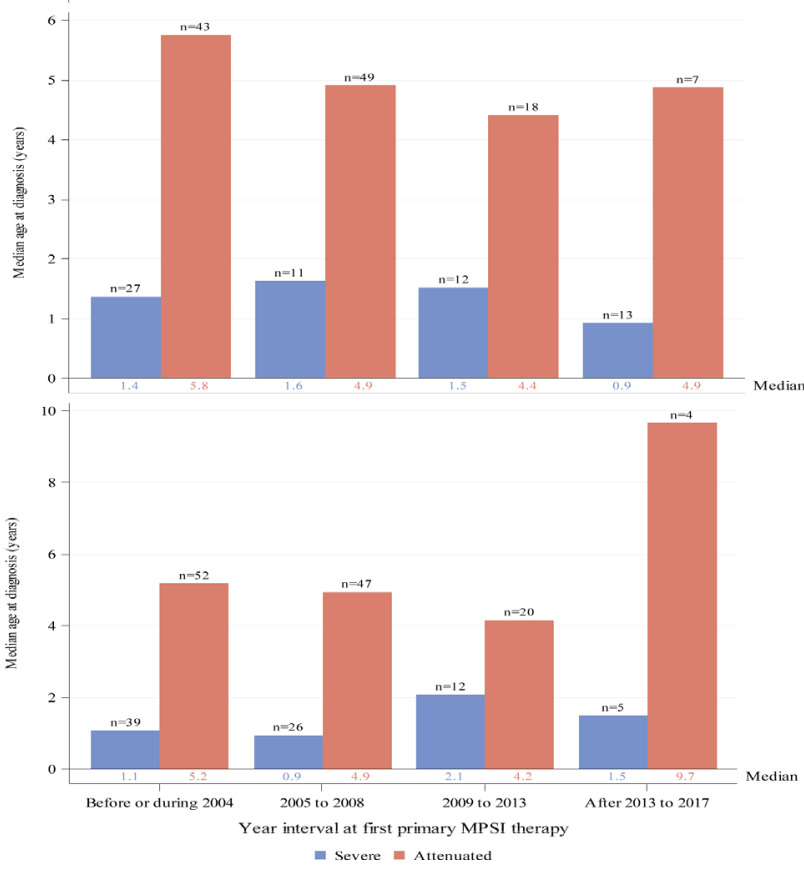

Figure 2 Median age in years at MPS I diagnosis by year interval for individuals with severe (blue) or attenuated (red) MPS I receiving ERT as primary therapy. Global distribution is shown in panel (A). Regional distributions are shown for North America (B) and Europe (C). Individual medians are shown at the base of each bar and individual n's are shown above the bars. ERT, enzyme replacement therapy; MPS I, mucopolysaccharidosis type I.

treatment at the earlier time intervals for individuals in Europe (figure 4C) compared with North America (figure 4B). Durations were similar by region in the more recent time intervals.

The median durations from diagnosis to first HSCT for all individuals with severe MPS I were very short for all time intervals (figure 5). Medians (IQR) were 0.2 (0.1, 0.3), 0.1 (0.1, 0.3), $0.1(0,0.2)$ and $0.1(0,0.1)$ years for intervals before/during 2004, 2005-2008, 2009-2013 and after 2013 to 2017, respectively. When stratified by North American and European regions (not shown), results were similar to the global data.

\section{DISCUSSION}

First signs and symptoms in individuals with MPS I vary in type, severity and age of onset, ${ }^{182425}$ which can lead to significant diagnostic delays due to the non-specific nature of symptoms that are suggestive of other diseases. This is particularly true for individuals with attenuated MPS I. ${ }^{17-19} 24$ In a study of the diagnostic history of 60 individuals with attenuated MPS I from the USA, Europe and Latin America, participants reported that, on average, there was a 3 -year delay between first physician visit and diagnosis for attenuated MPS I, and 20\% had delays of 5 years or longer. ${ }^{20}$ These are similar to regional surveys of MPS I patients/caregivers conducted 
A

B

C
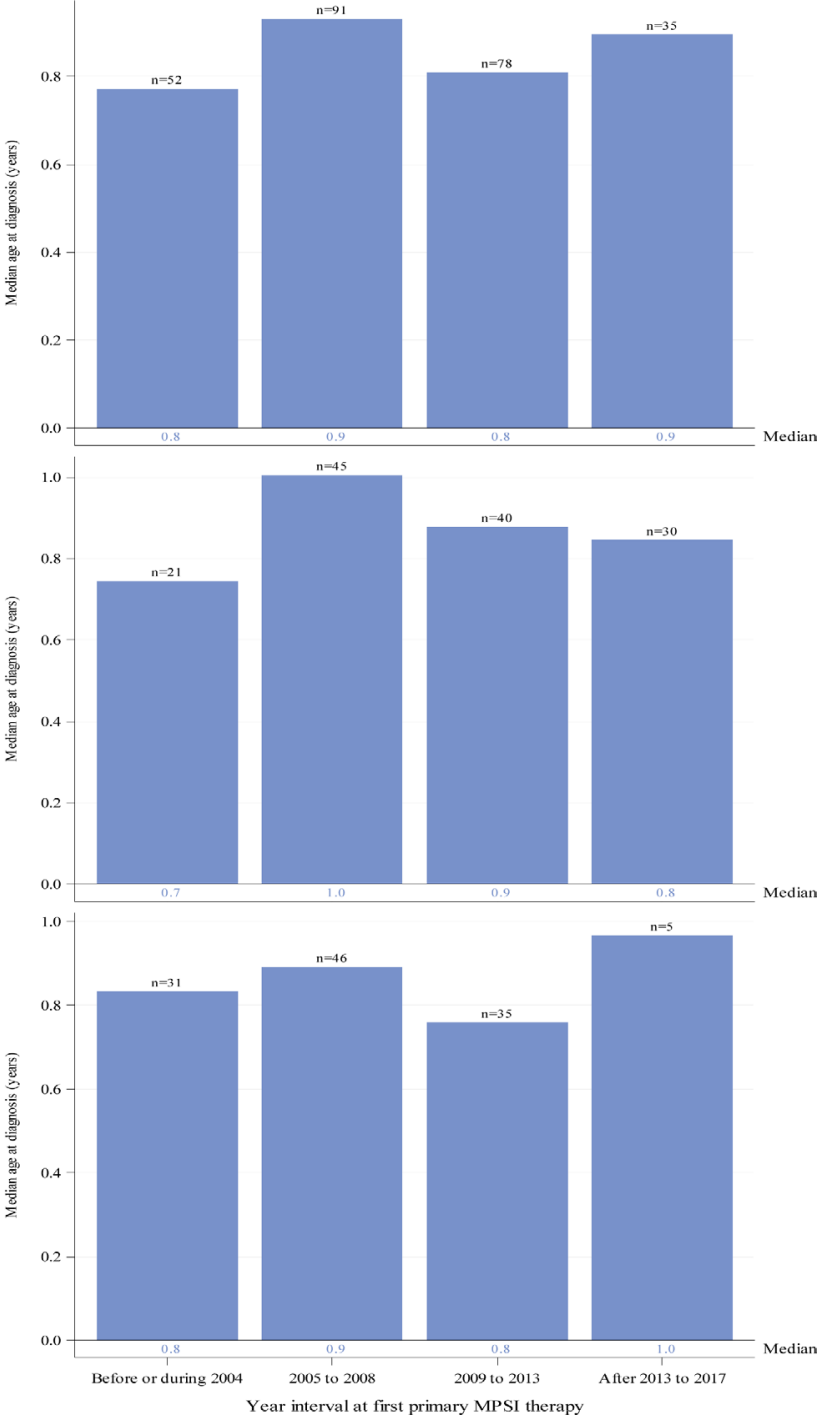

Figure 3 Median age in years at MPS I diagnosis by year interval for individuals with severe MPS I receiving HSCT as primary therapy. Global distribution is shown in panel (A). Regional distributions are shown for North America (B) and Europe (C). Individual medians are shown at the base of each bar and individual n's are shown above the bars. ERT, enzyme replacement therapy; HSCT, haematopoietic stem cell transplants; MPS I, mucopolysaccharidosis type I.

in the USA, Latin America and the Netherlands. ${ }^{21-23}$ Referrals to rheumatologists, orthopaedists, pulmonologists and gastroenterologists are common once children with MPS I present to paediatricians. ${ }^{26}$ Regardless of disease severity or initial symptoms, a mean of five specialists were consulted before receiving a correct diagnosis, highlighting the continued need for awareness education within the medical community. ${ }^{20}$

Our analyses involve a large dataset of diagnosis and treatment trends over time for individuals with MPS I. Time intervals for analysis began with approval of laronidase ERT by the FDA and EMA in 2003. We found that delays in diagnosis for individuals with attenuated MPS I have not improved over time. For patients not identified by newborn screening (NBS), algorithms to raise early clinical suspicion of MPS I based on the range of key signs and symptoms, ${ }^{26-29}$ are available for clinicians so that full diagnostic assessment ${ }^{30}$ of enzyme activity, substrate levels (ie, GAGs) and molecular analyses is conducted for appropriate
A

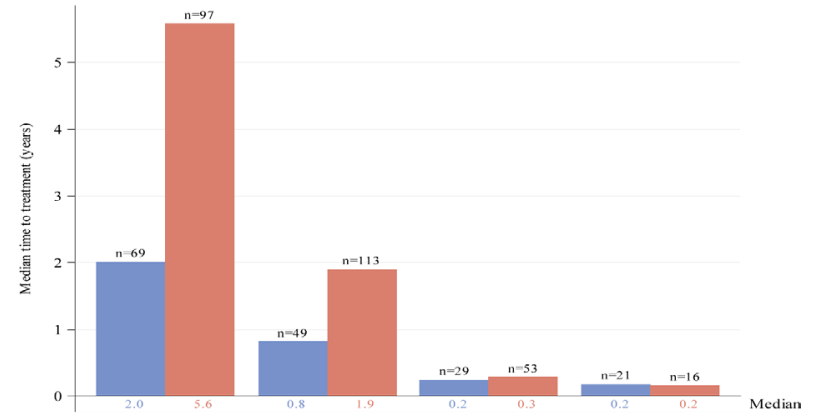

B

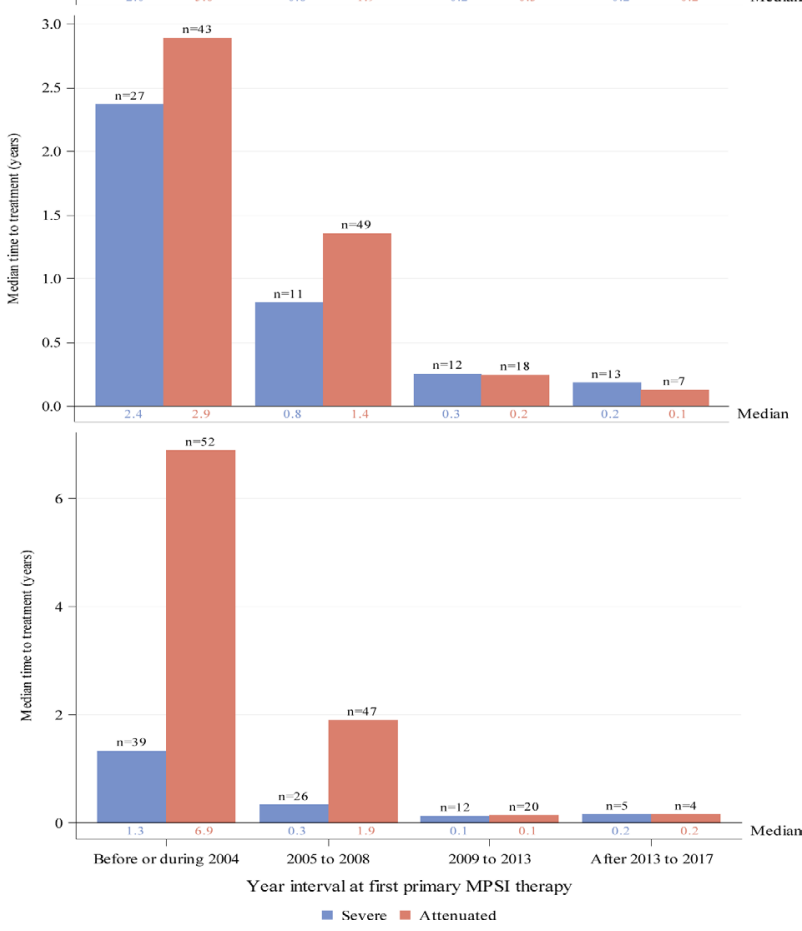

Figure 4 Median duration from diagnosis to first ERT treatment by year interval for individuals with severe (blue) or attenuated (red) MPS I receiving ERT as primary therapy. Global distribution is shown in panel (A). Regional distributions are shown for North America (B) and Europe (C). Individual medians are shown at the base of each bar and individual n's are shown at top of bars. ERT, enzyme replacement therapy; MPS I, mucopolysaccharidosis type I.

patients. ${ }^{30}$ It is worth noting that despite being a simple test, measurement of urinary GAGs is not available in all regions, and shipment of urine samples to reference laboratories may be difficult to arrange. In addition, urinary GAG levels may not be informative in young infants due to lack of standardisation of age-related reference ranges, ${ }^{31}$ The use of dried blood (DBS) for measurement of GAGs may provide useful information before another sample is requested. ${ }^{32}$

It is hoped that educational initiatives in combination with evolving NBS programmes worldwide, ${ }^{30} 31$ 33-36 will decrease time to MPS I diagnosis and treatment. NBS for MPS I have used either tandem mass spectrometry or digital microfluidic to measure IDUA enzyme activity in DBS. ${ }^{34} 36$ Follow-up of a positive NBS result (deficient IDUA activity) should include confirmatory IDUA enzyme activity in leucocytes, serum or plasma, urine GAG analysis and molecular analysis. ${ }^{31}$ The presence of IDUA pseudodeficiency alleles (benign variants) complicates NBS for MPS I since the pseudodeficiency alleles are more common than pathogenic IDUA variants. ${ }^{37}$ Pseudodeficiency is decreased IDUA activity with no evidence of GAG storage. 


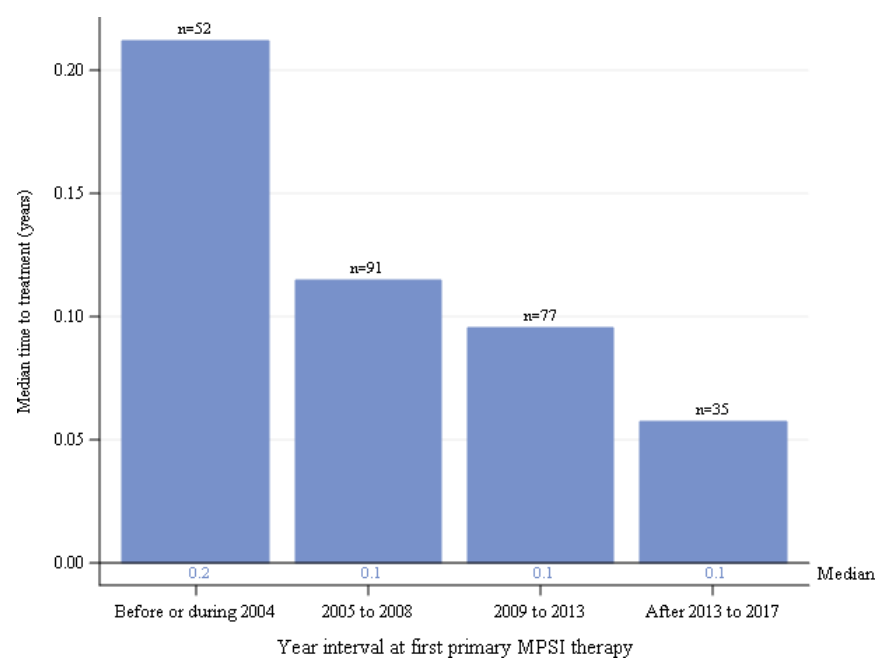

Figure 5 Median duration from diagnosis to first HSCT treatment by year interval for individuals with severe MPS I receiving HSCT as primary therapy. Global distribution is shown. HSCT, haematopoietic stem cell transplants; MPS I, mucopolysaccharidosis type I.

Second-tier testing measuring either GAG levels or sequencing of the IDUA gene using the original DBS has been used to reduce the NBS false-positive rate and the follow-up burden. ${ }^{32}$ 36-38 The MPS I phenotype can be predicted based on genotyping in the majority of cases, ${ }^{39}$ and early treatment initiated based on predicted phenotype for severe MPS I (HSCT with or without peritransplant ERT) or intravenous ERT for attenuated phenotypes. ${ }^{31}$ However, infants with abnormal NBS whose phenotypes cannot be positively predicted (ie, IDUA variants of unknown significance) require careful clinical and biochemical monitoring in order to identify appropriate treatment. ${ }^{313536}$

In general, individuals with severe MPS I are diagnosed at a younger age than those with attenuated disease, in part due to the more pronounced somatic symptoms. Our results mirror previous reports of MPS I Registry data, ${ }^{17}$ showing that this trend has not changed with time. Early diagnosis of severe MPS I is important since HSCT is indicated before 2 years of age, and early treatment is associated with better outcomes. ${ }^{14} 1640$ MPS I management decisions following positive NBS depend on the integration of biochemical, molecular and clinical assessments. ${ }^{3031}$ In the case of a patient identified with severe MPS I based on the presence of two pathogenic IDUA variants that predict this phenotype, the patient is referred for HSCT and potentially early peri-HSCT ERT. ${ }^{31}$ In accordance with current guidelines, HSCT should be ideally performed before 1 year of age, and typically not after 2.5 years, although the upper age limits vary by geographical region. ${ }^{40} 41$ A 10 year follow-up study showed that peritransplant ERT is associated with good outcomes in patients with severe MPS I. ${ }^{10}$

Interestingly, for individuals in the MPS I Registry with severe MPS I for whom ERT was the primary therapy, age at diagnosis was older than those with severe MPS I receiving HSCT as primary therapy by up to 1.1 years depending on treatment interval. This may reflect differences in onset of key somatic symptoms in some patients that are hallmarks of severe MPS I and drive diagnosis or differences in regional access to healthcare. We should also consider that patients diagnosed after the age of 2.0 years may not have been eligible for HSCT according to treatment guidelines, driving the patients to ERT monotherapy. ${ }^{42}$ HSCT is not always the primary treatment option for patients with severe MPS I. In some countries, the logistical and clinical challenges associated with HSCT remain high and families may decide not to take the risk. In addition to age restrictions, there are individuals with severe MPS I for whom HSCT is not a treatment option due to compromised physical state or lack of a suitable donor. Thus, patients with severe MPS I may still receive only ERT, although outcomes with ERT monotherapy in patients with severe MPS I are inferior to those obtained with early HSCT. ${ }^{42}$

While multiple studies have investigated delays between symptom onset and MPS I diagnosis, there is much less known regarding time between diagnosis and treatment onset. The duration from diagnosis to first treatment with ERT has improved substantially over time since introduction of laronidase ERT in 2003, from a median of over 5 years to less than 2 months. Early treatment with ERT has been shown to considerably improve patient outcomes during long-term therapy. ${ }^{12} 1315$ We anticipate that NBS programmes will result in decreased times to diagnosis and treatment with implementation and evolution of best management practices. ${ }^{31} 3542$

\section{Strengths and weaknesses of study}

As with all observational studies, there exists the potential for ascertainment and reporting biases due in part to the voluntary and observational nature of the MPS I Registry, which may impact the generalisability of the findings. The analyses included registry participants that subsequently died after data were captured on age at diagnosis and time to primary treatment. While a potential for bias is introduced by including patients that died who ultimately may have had severe disease, the bias would likely result in shorter times to diagnosis. The limitations of this analysis include the small numbers of individuals due to the nature of rare diseases, as well as limited data available in some regions. In particular, the smaller number of individuals from Latin America and Asia limit the comparison of these regions' trends with those for North America and Europe and make it difficult to compare factors such as healthcare system practices that may affect age at diagnosis and time to treatment. Specifically, there are significant differences in the healthcare practices of individual countries with respect to reimbursement of ERT and HSCT that may impact time to treatment, as well as challenges that patients and their families face in travelling to specialty centres for ERT infusions and/or transplants.

\section{CONCLUSION}

Early diagnosis is crucial for the best therapeutic outcomes with both ERT and HSCT to reduce disease progression before irreversible organ and tissue damage occur. The present study demonstrates that diagnosis of MPS I is delayed. However, time to treatment initiation, once individuals have been diagnosed, has improved substantially in the last 15 years for patients with either severe or attenuated MPS I. Efforts to improve early diagnosis are needed to ensure that patients receive appropriate treatment at the optimal time. These data illustrate that across regions, improvement in MPS I awareness is still needed.

Contributors RG contributed to the conception and design of the manuscript, analysis and interpretation of the data, drafted and revised the manuscript, approved the final version and is accountable for all aspects of the manuscript. NM contributed to interpretation of the data, revised the manuscript, approved the final version and is accountable for all aspects of the manuscript. MD contributed to interpretation of the data and revision of the manuscript, approved the final version and is accountable for all aspects of the manuscript. HK contributed to the conception and design of the manuscript, analysis and interpretation of the data, drafted and revised the manuscript, approved the final version and is accountable for all aspects of the manuscript. JM contributed to the conception and design of the manuscript, analysis 
and interpretation of the data, drafted and revised the manuscript, approved the final version and is accountable for all aspects of the manuscript.

Funding The study was funded by Sanofi Genzyme, Cambridge, Massachusetts, USA. Patrice C Ferriola, PhD, provided medical writing and editing assistance and was funded by Sanofi Genzyme.

Competing interests $\mathrm{RG}$ has received consultant/speaker honoraria/fees and/ or served on advisory boards for Actelion, Amicus, Biomarin, Inventiva, RegenxBio, Sanofi Genzyme, Sobi, Takeda and Ultragenyx, conducts contracted research for BioMarin, JCR, Lysogene, RegenxBio, Sanofi Genzyme, Takeda, and Ultragenyx and is a member of the International Board of Advisors of the MPS I Registry. NM is a consultant for Biomarin, Sanofi Genzyme, Lysogene, Shire and Sobi, has received grants/research support from Amicus, Biomarin, Sanofi Genzyme and Shire and has received honoraria and/or travel grants from Actelion, Amicus, BioMarin, Chiesi Farmaceutici S.p.A., Sanofi Genzyme and Shire. HK was an employee of Sanofi Genzyme at the time of the study.MD has received consultant/speaker honoraria and/or travel grants from Biomarin, Sanofi Genzyme, Regenxbio, Bluebird Bio, Ultragenyx. JM received consultant/speaker honoraria/fees and served on advisory boards for BioMarin, Denali, Eloxx, RegenxBio, Sangamo, Sanofi Genzyme and Shire, is a PI for a Phase I/II and Phase II/III intrathecal enzyme replacement clinical trials for MPS II and a Phase I/II gene editing clinical trial for MPS II and is a member of the International Board of Advisors of the MPS I Registry.

Patient consent for publication Not required.

Provenance and peer review Not commissioned; externally peer reviewed.

Data availability statement Data may be obtained from a third party and are not publicly available. The data that support the findings of this study can be requested by MPS I Registry participants through a MPS I Registry Data Analyses Request form. The data are not publicly available due to privacy or ethical restrictions. For additional information, please contact rarediseaseregistries@sanofi. com.

Open access This is an open access article distributed in accordance with the Creative Commons Attribution Non Commercial (CC BY-NC 4.0) license, which permits others to distribute, remix, adapt, build upon this work non-commercially, and license their derivative works on different terms, provided the original work is properly cited, appropriate credit is given, any changes made indicated, and the use is non-commercial. See: http://creativecommons.org/licenses/by-nc/4.0/.

ORCID iD

Roberto Giugliani http://orcid.org/0000-0001-9655-3686

\section{REFERENCES}

1 Muenzer J. Overview of the mucopolysaccharidoses. Rheumatology 2011;50 Suppl 5:v4-12.

2 Moore D, Connock MJ, Wraith E, et al. The prevalence of and survival in mucopolysaccharidosis I: Hurler, Hurler-Scheie and Scheie syndromes in the UK. Orphanet J Rare Dis 2008;3:24-30.

3 Federhen A, Pasqualim G, de Freitas TF, et al. Estimated birth prevalence of mucopolysaccharidoses in Brazil. Am J Med Genet A 2020;182:469-83.

4 Neufeld E, Muenzer J. The mucopolysaccharidoses. In: Schriver C, Beaudet A, Sly W, et al, eds. The metabolic and molecular basis of inherited disease. New York: McGraw Hill, 2001: p. 3421-52.

5 Thomas JA, Beck M, Clarke JTR, et al. Childhood onset of Scheie syndrome, the attenuated form of mucopolysaccharidosis I. J Inherit Metab Dis 2010;33:421-7.

6 Tolar J, Orchard PJ. Alpha-L-Iduronidase therapy for mucopolysaccharidosis type I. Biologics 2008;2:743-51.

7 Muenzer J, Wraith JE, Clarke LA, et al. Mucopolysaccharidosis I: management and treatment guidelines. Pediatrics 2009;123:19-29.

8 de Ru MH, Boelens JJ, Das AM, et al. Enzyme replacement therapy and/ or hematopoietic stem cell transplantation at diagnosis in patients with mucopolysaccharidosis type I: results of a European consensus procedure. Orphanet $J$ Rare Dis 2011;6:55-62.

9 Wraith JE. The first 5 years of clinical experience with laronidase enzyme replacement therapy for mucopolysaccharidosis I. Expert Opin Pharmacother 2005;6:489-506.

10 Ghosh A, Miller W, Orchard PJ, et al. Enzyme replacement therapy prior to haematopoietic stem cell transplantation in Mucopolysaccharidosis Type I: 10 year combined experience of 2 centres. Mol Genet Metab 2016;117:373-7.

11 Giugliani R. Mucopolysacccharidoses: from understanding to treatment, a century of discoveries. Genet Mol Biol 2012;35:924-31.

12 Laraway S, Breen C, Mercer J, et al. Does early use of enzyme replacement therapy alter the natural history of mucopolysaccharidosis I? Experience in three siblings. Mol Genet Metab 2013;109:315-6.

13 Muenzer J. Early initiation of enzyme replacement therapy for the mucopolysaccharidoses. Mol Genet Metab 2014;111:63-72.
14 Poe MD, Chagnon SL, Escolar ML. Early treatment is associated with improved cognition in Hurler syndrome. Ann Neurol 2014;76:747-53.

15 Gabrielli O, Clarke LA, Bruni S, et al. Enzyme-Replacement therapy in a 5-monthold boy with attenuated presymptomatic MPS I: 5-year follow-up. Pediatrics 2010;125:e183-7.

16 Aldenhoven M, Wynn RF, Orchard PJ, et al. Long-Term outcome of Hurler syndrome patients after hematopoietic cell transplantation: an international multicenter study. Blood 2015; 125:2164-72.

17 D'Aco K, Underhill L, Rangachari L, et al. Diagnosis and treatment trends in mucopolysaccharidosis I: findings from the MPS I Registry. Eur J Pediatr 2012;171:911-9.

18 Vijay S, Wraith JE. Clinical presentation and follow-up of patients with the attenuated phenotype of mucopolysaccharidosis type I. Acta Paediatr 2005;94:872-7.

19 Beck M, Arn P, Giugliani R, et al. The natural history of MPS I: global perspectives from the MPS I Registry. Genet Med 2014;16:759-65.

20 Bruni S, Lavery C, Broomfield A. The diagnostic journey of patients with mucopolysaccharidosis I: a real-world survey of patient and physician experiences. Mol Genet Metab Rep 2016;8:67-73.

21 de Ru MH, Bouwman MG, Wijburg FA, et al. Experiences of parents and patients with the timing of Mucopolysaccharidosis type I (MPS I) diagnoses and its relevance to the ethical debate on newborn screening. Mol Genet Metab 2012;107:501-7.

22 Kuiper G-A, Meijer OLM, Langereis EJ, et al. Failure to shorten the diagnostic delay in two ultra-orphan diseases (mucopolysaccharidosis types I and III): potential causes and implications. Orphanet J Rare Dis 2018:13:2.

23 Vieira T, Schwartz I, Muñoz V, et al. Mucopolysaccharidoses in Brazil: what happens from birth to biochemical diagnosis? Am J Med Genet A 2008;146A:1741-7.

24 Pastores GM, Arn P, Beck M, et al. The MPS I registry: design, methodology, and early findings of a global disease Registry for monitoring patients with Mucopolysaccharidosis Type I. Mol Genet Metab 2007;91:37-47.

25 Cleary MA, Wraith JE. The presenting features of mucopolysaccharidosis type IH (Hurler syndrome). Acta Paediatr 1995;84:337-9.

26 Cimaz R, Coppa GV, Koné-Paut l, et al. Joint contractures in the absence of inflammation may indicate mucopolysaccharidosis. Pediatr Rheumatol Online J 2009;7:18-25

27 Tylki-Szymańska A, De Meirleir L, Di Rocco M, et al. Easy-To-Use algorithm would provide faster diagnoses for mucopolysaccharidosis type I and enable patients to receive earlier treatment. Acta Paediatr 2018;107:1402-8.

28 Guffon N, Journeau P, Brassier A, et al. Growth impairment and limited range of joint motion in children should raise suspicion of an attenuated form of mucopolysaccharidosis: expert opinion. Eur J Pediatr 2019;178:593-603.

29 Martins AM, Lindstrom K, Kyosen SO, et al. Short stature as a presenting symptom of attenuated Mucopolysaccharidosis type I: case report and clinical insights. BMC Endocr Disord 2018;18:83.

30 Kubaski F, de Oliveira Poswar F, Michelin-Tirelli K, et al. Diagnosis of Mucopolysaccharidoses. Diagnostics 2020;10:172.

31 Clarke LA, Atherton AM, Burton BK, et al. Mucopolysaccharidosis Type I newborn screening: best practices for diagnosis and management. J Pediatr 2017;182:363-70.

32 Kubaski F, Suzuki Y, Orii K, et al. Glycosaminoglycan levels in dried blood spots of patients with mucopolysaccharidoses and mucolipidoses. Mol Genet Metab 2017:120:247-54.

33 Lin S-P, Lin H-Y, Wang T-J, et al. A pilot newborn screening program for Mucopolysaccharidosis type I in Taiwan. Orphanet J Rare Dis 2013;8:147.

34 Hopkins PV, Campbell C, Klug T, et al. Lysosomal storage disorder screening implementation: findings from the first six months of full population pilot testing in Missouri. J Pediatr 2015;166:172-7.

35 Parini R, Broomfield A, Cleary MA, et al. International working group identifies need for newborn screening for mucopolysaccharidosis type I but states that existing hurdles must be overcome. Acta Paediatr 2018;107:2059-65.

36 Donati MA, Pasquini E, Spada M, et al. Newborn screening in mucopolysaccharidoses. Ital J Pediatr 2018;44:126.

37 Taylor JL, Clinard K, Powell CM, et al. The North Carolina experience with Mucopolysaccharidosis Type I newborn screening. J Pediatr 2019;211:193-200.

38 Polo G, Gueraldi D, Giuliani A, et al. The combined use of enzyme activity and metabolite assays as a strategy for newborn screening of mucopolysaccharidosis type I. Clin Chem Lab Med 2020. doi:10.1515/cclm-2020-0064. [Epub ahead of print: 20 May 2020].

39 Clarke LA, Giugliani R, Guffon N, et al. Genotype-Phenotype relationships in mucopolysaccharidosis type I (MPS I): insights from the International MPS I registry. Clin Genet 2019;96:281-9.

40 Aldenhoven $\mathrm{M}$, Jones SA, Bonney D, et al. Hematopoietic cell transplantation for mucopolysaccharidosis patients is safe and effective: results after implementation of international guidelines. Biol Blood Marrow Transplant 2015;21:1106-9.

41 Parini R, Deodato F, Di Rocco M, et al. Open issues in Mucopolysaccharidosis type I-Hurler. Orphanet J Rare Dis 2017;12:112.

42 Eisengart JB, Rudser KD, Xue Y, et al. Long-Term outcomes of systemic therapies for Hurler syndrome: an international multicenter comparison. Genet Med 2018:20:1423-9. 